This item was submitted to Loughborough's Research Repository by the author.

Items in Figshare are protected by copyright, with all rights reserved, unless otherwise indicated.

\title{
On the dephasing time of the chiral metal
}

PLEASE CITE THE PUBLISHED VERSION

http://dx.doi.org/10.1209/epl/i2001-00124-7

\section{PUBLISHER}

Institute of Physics (IOP) / @ EDP Sciences

VERSION

SMUR (Submitted Manuscript Under Review)

LICENCE

CC BY-NC-ND 4.0

\section{REPOSITORY RECORD}

Betouras, Joseph J.. 2019. "On the Dephasing Time of the Chiral Metal". figshare. https://hdl.handle.net/2134/12777. 
This item was submitted to Loughborough's Institutional Repository (https://dspace.lboro.ac.uk/) by the author and is made available under the following Creative Commons Licence conditions.

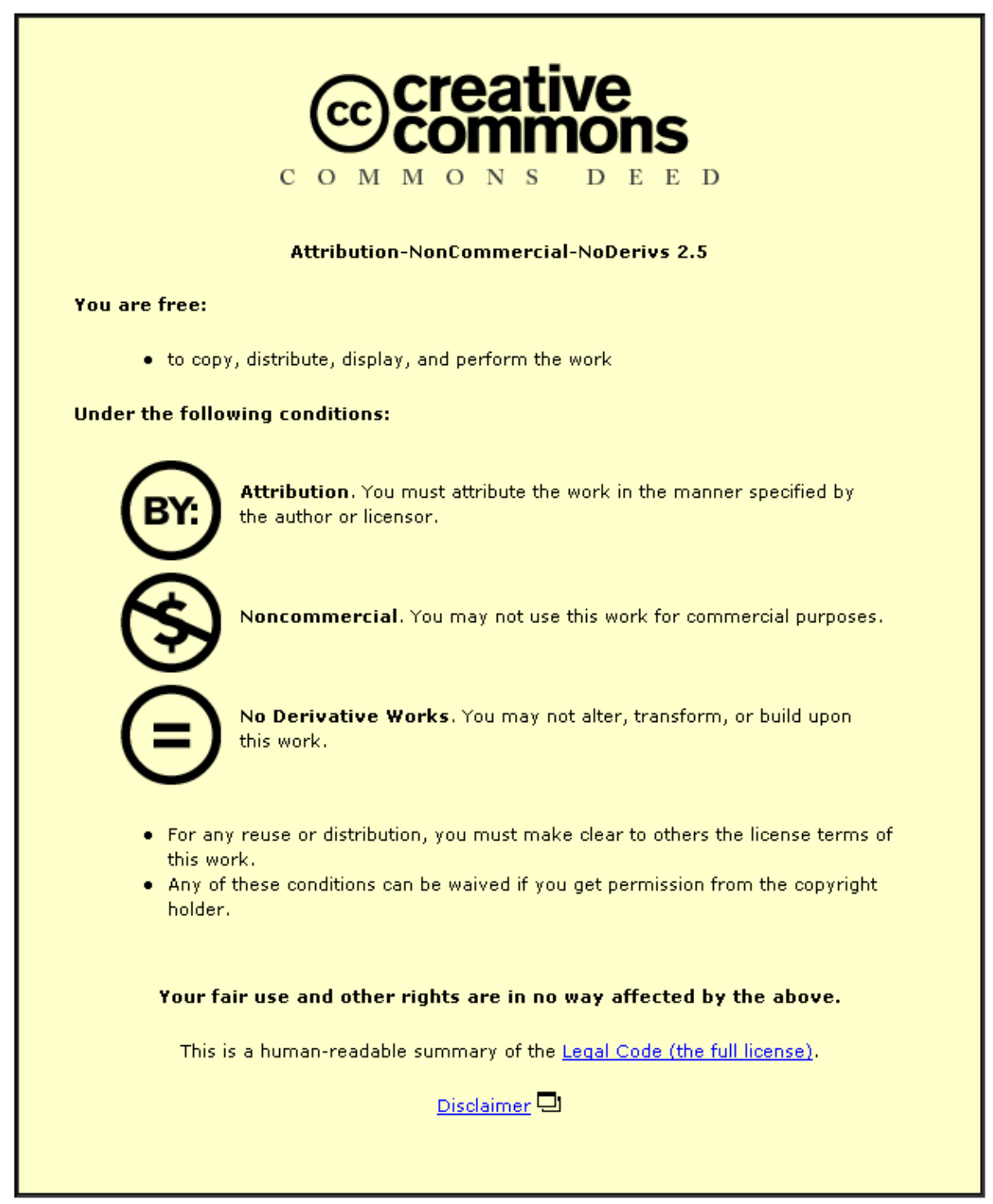

For the full text of this licence, please go to: http://creativecommons.org/licenses/by-nc-nd/2.5/ 
EUROPHYSICS LETTERS

Europhys. Lett., (), pp. ()

\title{
On the dephasing time of the chiral metal
}

\author{
J.J. Betouras \\ Department of Physics, Theoretical Physics, University of Oxford, \\ 1 Keble Road, Oxford, OX1 3NP, U.K. \\ (received ; accepted ) \\ PACS. 73.23.-b - Mesoscopic systems. \\ PACS. 73.20.Dx - Electron states in low-dimensional structures (superlattices, quantum well \\ structures and multilayers). \\ PACS. 73.40.Hm- Quantum Hall effect (integer and fractional).
}

\begin{abstract}
In the low-dimensional disordered systems the dephasing time and the inelastic scattering (out-scattering) time are in general different. We show that in the case of the twodimensional chiral metal which is formed at the surface of a layered three dimensional system, which is exhibiting the integer quantum Hall effect these two quantities are essentially the same and their temperature-dependence is $T^{-\frac{3}{2}}$. In particular we show that the results obtained using the diagramatic technique and the phase uncertainty approach introduced by A. Stern et al. (Phys. Rev. A 41, 3436 (1990)) for the out-scattering and the dephasing time respectively, coincide. We furthermore consider these quantities in the case of the three-dimensional chiral metal, where similar conclusions are reached.
\end{abstract}

Recently there has been much interest in the properties of the states formed at the surface of a three dimensional (3D) structure which is constructed by stacking together parallel twodimensional systems which exhibit the integer quantum Hall effect. The first study in this direction, by Störmer and collaborators [1] concluded that strict two-dimensionality is not required for the observation of the quantum Hall Effect and showed that the conductivity in the direction of the field $\sigma_{z z}$ tends to zero in the bulk, as the temperature T approaches zero.

Subsequent theoretical studies, first by Chalker and Dohmen 2] and later by Balents and Fisher [3] were focused on the nature of the two dimensional states at the surface of the multilayered quantum Hall systems. In particular, the former demonstrated the existence of the quantum Hall regime in the layered 3D conductors in strong magnetic field as well as the surface states and predicted a metallic behaviour on the $z$-axis. The latter, emphasized the absence of any localization effects and mentioned that there are no singularities in the density of states (DOS). The basic consequence of the absense of backscattering due to the unidirectional electronic motion, is the Fermi liquid behavior which is retained in the presence of interactions as opposed to the Luttinger liquid behavior in the interacting one-dimensional electronic systems.

An experiment by Druist et al. [A on GaAs $/ \mathrm{Al}_{0.1} \mathrm{Ga}_{0.9} \mathrm{As}$ multilayers allowed the observation of electrons that are transported through the surface states, and indicated the possibility

Typeset using EURO-TEX 
of the suppression of localization effects. In this experiment, the conductance $g_{z z}$ approached a non-zero constant at very low temperatures in the quantum Hall regime, and was proportional to the circumference of the sample and not to the area of the layers. This constituted a convincing evidence that electrons are transported via the surface and not via the bulk.

The next phase of the experiments was to measure the conductance fluctuations and to provide information on microscopic quantities [7]. In addition to that, conduction by surface states was considered for the interpretation of experiments on bulk quantum Hall effect in organic conductors [6] and an inorganic quasi-two dimensional conductor [8].

In a very recent theoretical study [5] the screened Coulomb interaction was taken into account in detail. Among other quantities, the inelastic scattering rate (out- scattering rate in the mesoscopic physics language) $\gamma=\hbar / \tau_{e e}$ was calculated using the diagrammatic technique [10, 11, 13] and was found to vary as $T^{3 / 2}$. In general in the low dimensional interacting and disordered electron systems the out-scattering time is different from the dephasing time $\tau_{\phi}$. The out-scattering time is the quantity that appears in the expression of the diffuson when the interaction effects are taken into account and therefore it can be measured indirectly through the measurements of the amplitude of the conductance fluctuations 14, 5]. The dephasing time, on the other hand, is the time it takes for the electrons to lose their phase coherence due to their interactions with the environment and is the physical quantity in low-dimensional disordered systems. The purpose of this letter is to show explicitly that in the system under consideration the two times coincide and this is a special feature of the chirality of the system as we will discuss. In doing that we also compare two different established calculational techniques.

The Hamiltonian $H$ of the non-interacting system, acting on a wavefunction $\psi_{n}(x)$ where $n$ labels the layer and $x$ is the chiral direction, is given by [2, 9]:

$$
(H \psi)_{n}(x)=-i v \hbar \partial_{x} \psi_{n}(x)-t\left[\psi_{n+1}(x)+\psi_{n-1}(x)\right]+V_{n}(x) \psi_{n}(x),
$$

where $v$ is the chiral velocity, $t$ is the interlayer tunneling energy, $a$ is the interlayer spacing and $V_{n}(x)$ is a random potential arising from impurities and surface roughness. This random potential is chosen to be Gaussian distributed with short-range correlations : $\left\langle V_{n}(x)\right\rangle=0$ and $\left\langle V_{n}(x) V_{m}\left(x^{\prime}\right)\right\rangle=\Delta \delta_{n m} \delta\left(x-x^{\prime}\right)$. The diffusion constant is $D=2(a t)^{2} v / \Delta$. Then the disorder averaged, retarded one-particle Green's function $G^{R}(\omega ; \mathbf{k})$ in real frequency and momentum space and also the diffuson at small momenta $K(\omega, \mathbf{k})$ are given by :

$$
\begin{aligned}
G^{R}(\omega ; \mathbf{k}) & =\frac{1}{\left[\omega+i \Delta /(2 \hbar v)-\left(\hbar v k_{x}-2 t \cos \left(k_{z} a\right)\right]\right.} \\
K(\omega, \mathbf{k}) & =\frac{1}{(\hbar v)^{-1}\left[\hbar D k_{z}{ }^{2}-i\left(\omega+\hbar v k_{x}\right)\right]}
\end{aligned}
$$

The screened Coulomb interaction, in Matsubara formalism and in Random Phase Approximation (RPA), reads :

$$
U_{\text {eff }}\left(i \Omega_{n}, \mathbf{q}\right)=U^{0}(\mathbf{q}) / \epsilon=U^{0}(\mathbf{q}) /\left(1+U^{0}(\mathbf{q}) \Pi\left(i \Omega_{n}, \mathbf{q}\right)\right)
$$

where $\Omega_{n}=2 \pi k_{B} T$ is the Matsubara frequency, $U^{0}(\mathbf{q})=2 \pi e^{2} / q$ is the bare Coulomb interaction in $2 \mathrm{D}, \epsilon$ is the dielectric constant and $\Pi(i \Omega, \mathbf{q})$ is the polarization which in both the RPA and the hydrodynamic approaches [5] is :

$$
\Pi\left(i \Omega_{n}, \mathbf{q}\right)=n \frac{-i v q_{x} \operatorname{sgn}\left(\Omega_{n}\right)+D q_{z}^{2}}{\left|\Omega_{n}\right|-i v q_{x} \operatorname{sgn}\left(\Omega_{n}\right)+D q_{z}^{2}}
$$




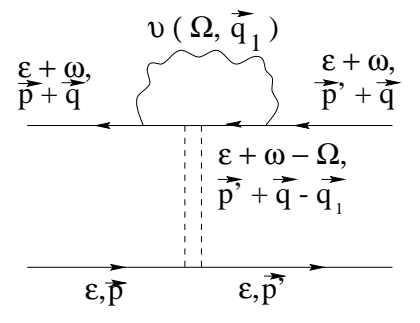

(i)

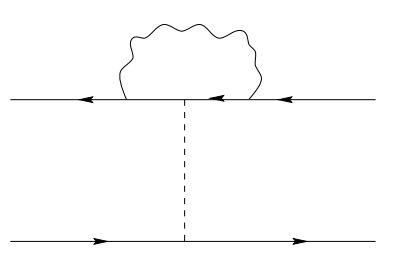

(iii)

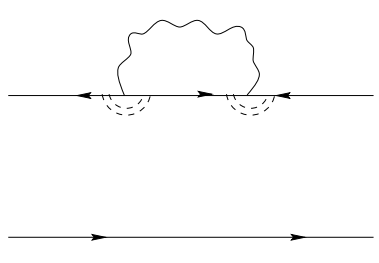

(ii)

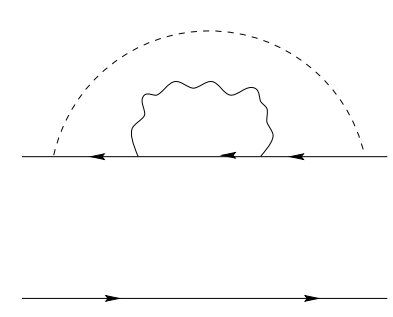

(iv)

Fig. 1. - The relevant diagrams for the calculation of the interaction block I. Straight lines correspond to electron propagators, wiggly lines to Coulomb interactions, double dashed lines to diffusons and single dashed lines to single impurity scattering.

with $n=1 / h v a$ being the density of states. In order to calculate $\gamma$ the interaction block technique can be used [13, 11, 5]:

$$
\gamma=-\frac{1}{\pi n \tau^{2}} I
$$

where I is the interaction block. The relevant diagrams, up to first order in the interaction, for this particular problem, are those shown in Fig. 1 together with their corresponding ones with the interaction in the lower Green's function. The contribution comes from diagrams which do not have interaction between different electronic lines.

In order to do the summation over Matsubara frequencies, the contours of integration in the complex $z$-plane $(z=i \Omega)$ for the two first diagrams include a branch-cut at $z=0$ and all of the four diagrams contain a "boundary" term at $z=i(\epsilon+\omega)$ or $z=i \epsilon$. Combining all the terms and simplifying it under the assumption that the major contribution comes from small momenta, we arrive at the expression for the out-scattering rate $\gamma$ :

$$
\begin{array}{r}
\gamma=\frac{1}{\pi \hbar n} \int \frac{d^{2} q}{(2 \pi)^{2}} \int_{0}^{+\infty} d x\left[\operatorname{coth}\left(x / 2 k_{B} T\right)-\tanh \left((x-\epsilon /) / 2 k_{B} T\right)\right] \times \\
\frac{1}{\left(x+\hbar v q_{x}\right)^{2}+\left(\hbar D q_{z}^{2}\right)^{2}} \frac{x\left(\hbar D q_{z}^{2}\right)^{2}}{\hbar^{2} v^{2} q_{x}{ }^{2}+\left(\hbar D q_{z}^{2}\right)^{2}}
\end{array}
$$

where the coth and tanh terms come from the Bose and the Fermi distribution function which appear in the $z=0$ branch-cut and the "boundary" terms respectively. The main contribution comes from the small energy regime and consequently the main term is the one with the coth. 
We can rescale the momenta and $\mathrm{x}$ to :

$$
x=k_{B} T X \quad v \hbar q_{x}=k_{B} T Q_{x} \quad \sqrt{\hbar D} q_{z}=\sqrt{k_{B} T} Q_{z}
$$

The temperature dependence can be then extracted in the limit of small temperatures and $\epsilon=0$, after performing the integration the full result is [5]:

$$
\gamma=1.5 \frac{a}{D^{1 / 2}}\left(\frac{k_{\mathrm{B}} T}{\hbar}\right)^{3 / 2}
$$

This $T$-dependence can be understood quantitatively in relation to the general argument that $\gamma \propto 1 / n_{d} L_{T}{ }^{d}$ where $d$ is the dimensionality of the system and $L_{T}$ is the characteristic thermal length which acts as a cut-off. In the case of the chiral metal the characteristic thermal lengths are different in the two dimensions due to the anistropic behavior. In that case $L_{T}{ }^{d}=L_{T, x} L_{T, z}$, and, since $L_{T, x} \propto(v / T)$ and $L_{T, z} \propto \sqrt{D / T}$ then the rate $\gamma \propto T^{3 / 2}$.

We now follow the general procedure for the computation of $\tau_{\phi}$ which was introduced in the pioneering work [16] by Stern, Aharonov and Imry (from now on in this paper referred as SAI). The basic physical picture which was put forward and analysed in this work (supplementing the important work of [15]) was the thought interference experiment of an electron which can choose a left or right path before interfering with itself. The description of the electron with the wave function $\psi(\mathbf{r})=\psi_{L}(\mathbf{r}) \otimes \psi_{R}(\mathbf{r})$ allows the independent interaction of each part (Left/Right) of the wave function with its environment. This leads naturally to dephasing and the key observation is that the phase uncertainty accumulated by the electron is given by twice the probability $P(t)$ that the environment alters its state due to the interaction with the electron. This allows for a quantitative treatment of the dephasing time; from the phase uncertainty we can get the information on the temperature dependence of the $\tau_{\phi}$ which is now defined as the time where $P\left(\tau_{\phi}\right) \simeq 1$. It is evident that this procedure does not allow for the accurate calculation of the prefactors.

If we consider up to second order terms in the interaction, then the probability is given by :

$$
\begin{array}{r}
P\left(t_{0}\right)=\sum_{|\alpha\rangle \neq|0\rangle} \int_{0}^{t_{0}} d t \int_{0}^{t_{0}} d t^{\prime}\left\langle 0\left|V\left(\mathbf{r}_{\mathbf{1}}(t), t\right)-V\left(\mathbf{r}_{\mathbf{2}}(t), t\right)\right| \alpha\right\rangle \\
\left\langle\alpha\left|V\left(\mathbf{r}_{\mathbf{1}}\left(t^{\prime}\right), t^{\prime}\right)-V\left(\mathbf{r}_{\mathbf{2}}\left(t^{\prime}\right), t^{\prime}\right)\right| 0\right\rangle
\end{array}
$$

where $V(\mathbf{r}, t)$ is the Coulomb interaction between the observed electron and the rest of the electrons which consist the environment, and $\mathbf{r}_{1}(t), \mathbf{r}_{2}(t)$ are the two different paths (of equal length) under consideration.

Then, using the fluctuation-dissipation theorem, $P$ is given by [17:

$P\left(t_{0}\right)=\frac{1}{\hbar} \int_{0}^{t_{0}} d t \int_{0}^{t_{0}} d t^{\prime} \int_{-\infty}^{\infty} d \omega \operatorname{coth}\left(\frac{\omega}{2 k_{B} T}\right) \int \frac{d^{2} q}{(2 \pi)^{2}} \frac{2 \pi e^{2}}{q} \operatorname{Im}\left(\frac{1}{\epsilon}\right) \exp \left[i \omega\left(t-t^{\prime}\right)+i \mathbf{q} \cdot\left[\mathbf{r}_{1}(t)-\mathbf{r}_{2}\left(t^{\prime}\right)\right]\right]$

The above expression contains only one of the four possible terms which arise when we take into account the phase fluctuations of the two paths $\left(<\left(\delta \phi_{1}-\delta \phi_{2}\right)^{2}>=<\left(\delta \phi_{1}\right)^{2}>+<\right.$ $\left.\left(\delta \phi_{2}\right)^{2}>-2<\delta \phi_{1} \delta \phi_{2}>\right)$. The other terms contain the phases :

$$
\exp \left(i \mathbf{q} \cdot\left[\mathbf{r}_{1}(t)-\mathbf{r}_{1}\left(t^{\prime}\right)\right]\right) \quad \exp \left(i \mathbf{q} \cdot\left[\mathbf{r}_{2}(t)-\mathbf{r}_{2}\left(t^{\prime}\right)\right]\right) \quad \exp \left(i \mathbf{q} \cdot\left[\mathbf{r}_{2}(t)-\mathbf{r}_{1}\left(t^{\prime}\right)\right]\right.
$$

For the chiral metal :

$$
\mathbf{q} \cdot\left[\mathbf{r}_{1}(t)-\mathbf{r}_{2}\left(t^{\prime}\right)\right]=q_{x} v\left(t-t^{\prime}\right)+q_{z}\left(z_{1}(t)-z_{2}\left(t^{\prime}\right)\right)
$$


$\operatorname{Im}(1 / \epsilon)$ is calculated using Eq.(4) and (5) and the factor coth restricts the integration to small frequencies $\left(-k_{B} T<\omega<k_{B} T\right)$ and can be expanded. Since the only $\omega$ dependence is retained in the exponential $\exp \left(i \omega\left(t-t^{\prime}\right)\right)$ in the limit of small $q$ (in fact the only mathematical assumption in the calculation is that $\left.1+2 \pi n e^{2} / q \simeq 2 \pi n e^{2} / q\right)$, and under the physical assumption that the duration of the experiment is longer than $1 / k_{B} T$, the integration over frequency can be well approximated by $\delta\left(t-t^{\prime}\right)$. The integration over $t^{\prime}$ then is trivial and the integration over $q_{x}$ can also be performed easily by a residue integration (note that the terms that come from the bare Coulomb interaction and contain $q$ drop out of the calculation under the mathematical assumption made above). If at this stage we take into account all four terms then the expression that remains to be evaluated is :

$$
P\left(t_{0}\right)=\frac{1}{n \hbar} \int_{0}^{t_{0}} d t k_{B} T \int d q_{z} \sin ^{2}\left[\frac{q_{z}}{2}\left(z_{1}(t)-z_{2}(t)\right)\right]
$$

The integrand of this expresion is identical to the integrand obtained in the case of the 3D, disordered, ordinary metal in spherical coordinates after performing the angular integrations 117 as we will comment below. The upper cut-off in the integration over $q_{z}$ is then $q_{z, \max }=$ $\sqrt{k_{B} T / D}=L_{T, z}{ }^{-1}$. In addition to that, and due to the diffusive nature of the motion in the $z$-direction, we can approximate $\left|z_{1}(t)-z_{2}(t)\right| \sim \sqrt{D t}$. Taking into account all the ingredients the final result is :

$$
P\left(t_{0}\right) \simeq t_{0} \frac{a}{\sqrt{D}}\left(k_{B} T\right)^{3 / 2}
$$

Since $P\left(t_{0}\right) \simeq O(1)$ then $\tau_{\phi} \propto\left(k_{B} T\right)^{-3 / 2}$, i.e. it exhibits the same $T$ - dependence as $\tau_{e e}$.

We recall that in the ordinary (non-chiral) $2 \mathrm{D}$ dirty metals the out-scattering time $\tau_{e e}^{-1} \sim$ $T|\log T|[10]$. The reason for the logarithmic correction is that the dominant contribution to the scattering comes from processes with small energy transfers. On the other hand these, processes are not the dominant ones in the calculation of the dephasing time and this fact leads to the difference in the temperature dependence of the two quantities. In the case of the chiral metal though the small energy transfer processes are not the dominant ones either for the dephasing or for the out-scattering time and therefore these quantities coincide.

If we consider the equivalent calculations for a 3D chiral metal (conceived as coupled chiral chains in two directions), where a ballistic motion occurs in one direction and a diffusive motion in the other two, then the polarization is modified to :

$$
\Pi\left(i \Omega_{n}, \vec{q}\right)=n \frac{-i v q_{x} \operatorname{sgn}\left(\Omega_{\mathrm{n}}\right)+D q_{y}{ }^{2}+D^{\prime} q_{z}{ }^{2}}{\left|\Omega_{n}\right|-i v q_{x} \operatorname{sgn}\left(\Omega_{\mathrm{n}}\right)+D q_{y}^{2}+D^{\prime} q_{z}{ }^{2}}
$$

where we assume different diffusion constants for the two non-chiral directions. Taking now into account that $U^{0}(\vec{q})=4 \pi e^{2} / q^{2}, \gamma$ becomes :

$$
\gamma=c \frac{a b}{\sqrt{D D^{\prime}}}\left(\frac{k_{B} T}{\hbar}\right)^{2}
$$

with $a$ and $b$ being the interchain distances in the two perpendicular directions to the chains and $c$ a constant of $O(1)$. The SAI approach then yields the same temperature dependence for the dephasing time for the same reasons as those described above. Note the accidental similarity in the temperature dependence of the dephasing rate of the $2 \mathrm{D}$ chiral metal with the $3 \mathrm{D}$ ordinary disordered metal as well as the similarity in the temperature dependence of the inelastic scattering rate of the 3D chiral metal with that of a $3 \mathrm{D}$ clean metal.

The conclusion of the presented work is that the novel electronic system (chiral metal) offers a chance to test the different approaches of calculating the characteristic times of a mesoscopic 
system. We considered the system in the regime where the inter-edge tunneling time $\tau_{\perp}$ satisfies the condition $\tau_{\perp} \ll \tau_{\phi}$. The dephasing time, although it has a transparent physical meaning, is difficult to be measured in the chiral metal but the temperature dependence of the out-scattering time can be extracted through the amplitude of the conductance fluctuations. We demonstrated that the two times have the same temperature dependence as a result of the subdominence of the small energy transfer processes. The general quantitative arguments given in 10] which can be formally represented by the interaction block technique are confirmed in this case and give identical results with the procedure introduced by SAI. The interplay of electron-electron interactions and disorder in this low-dimensional system does not lead to the same consequences as in the ordinary dirty metallic systems due to the absence of self-intersecting paths in the electronic motion.

The author is grateful to John Chalker for helpful discussions and careful reading of the manuscript. This work has been supported in part by the European Union under TMR FMBICT972304 and in part by EPSERC under grant No GR/J8327.

\section{REFERENCES}

[1] H. L. Störmer, J. P. Eisenstein, A. C. Gossard, W. Wiegmann and K. Baldwin, Phys. Rev. Lett. 56, 85 (1985); H. L. Störmer et al. Proceedings of the 18th International Conference on the Physics of Semiconductors, Stockholm, 1986, edited by O. Engstrom (World Scientific, Singapore, 1987), p. 385.

[2] J. T. Chalker and A. Dohmen, Phys. Rev. Lett. 75, 4496 (1995).

[3] L. Balents and M. P. A. Fisher, Phys. Rev. Lett. 76, 2782 (1996).

[4] D. P. Druist, P. J. Turley, K. D. Maranowski, E. G. Gwinn and A. C. Gossard, Phys. Rev. Lett. 80, 365 (1998).

[5] J. J. Betouras and J. T. Chalker, to appear in Phys. Rev. B 62 (2000); also cond-mat/0005151

[6] S. Hill et al, Phys. Rev. B 55, R4891 (1997); M. M. Honold et al, J. Phys. Cond. Matt. 9, L533 (1997); S. Uji et al, Phys. Rev. 60, 1650, (1999).

[7] D. P. Druist et al, Superlattices and Microstructures, 25, 181 (1999)

[8] S. Hill et al, Phys. Rev. B 58, 10778 (1998).

[9] J. T. Chalker and S. L. Sondhi, Phys. Rev. B 59, 4999 (1999).

[10] B. L. Altshuler and A. G. Aronov, in Electron - electron Interactions in Disordered Systems, edited by A. L. Efros and M. Pollak (North- Holland, Amsterdam, 1985), p. 1.

[11] H. Fukuyama and E. Abrahams, Phys. Rev. B 27, 5976 (1983).

[12] E. Abrahams, P. W. Anderson, P. A. Lee and T. V. Ramakrishnan, Phys. Rev. B 24, 6783 (1981).

[13] Ya. M. Blanter, Phys. Rev. B 54, 12807 (1996).

[14] P. A. Lee, A. D. Stone and H. Fukuyama, Phys. Rev. B 351039 (1987) ; A. D. Stone and P. A. Lee, Phys. Rev. Lett. 54, 1196 (1985)

[15] B. L. Altshuler, A. G. Aronov, D. E. Khmelnitsky J. Phys. C 15, 7367 (1982)

[16] A. Stern, Y. Aharonov, Y. Imry Phys. Rev. A 41, 3436 (1990) ; also an application to quantum dots is in U. Sivan, Y. Imry and A. G. Aronov Europhys. Lett. 28, 115 (1994)

[17] Y. Imry, Introduction to Mesoscopic Physics, Oxford University Press (1997) 